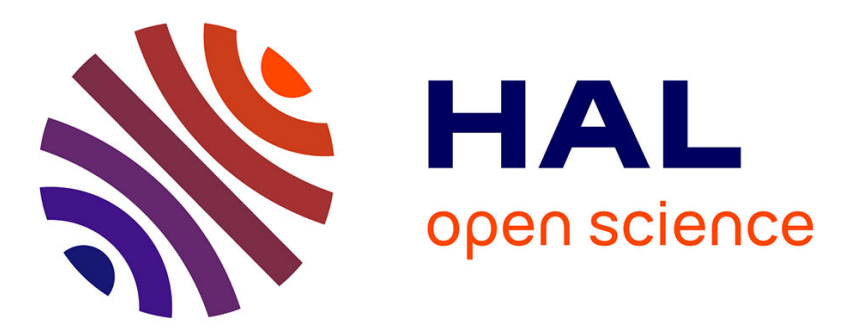

\title{
Review of spectral reflectance models for halftone prints: Principles, Calibration, and prediction accuracy
}

\author{
Mathieu Hébert, Roger D. Hersch
}

\section{To cite this version:}

Mathieu Hébert, Roger D. Hersch. Review of spectral reflectance models for halftone prints: Principles, Calibration, and prediction accuracy. Color Research and Application, 2014, 0, pp.1. 10.1002/col.21907 . hal-01076364

\section{HAL Id: hal-01076364 https://hal.science/hal-01076364}

Submitted on 21 Oct 2014

HAL is a multi-disciplinary open access archive for the deposit and dissemination of scientific research documents, whether they are published or not. The documents may come from teaching and research institutions in France or abroad, or from public or private research centers.
L'archive ouverte pluridisciplinaire HAL, est destinée au dépôt et à la diffusion de documents scientifiques de niveau recherche, publiés ou non, émanant des établissements d'enseignement et de recherche français ou étrangers, des laboratoires publics ou privés. 


\title{
Review of spectral reflectance models for halftone prints: principles, calibration, and prediction accuracy ${ }^{1}$
}

\author{
Mathieu Hébert ${ }^{\mathrm{a}, \mathrm{b}}$, Roger D. Hersch ${ }^{\mathrm{c}}$ \\ ${ }^{a}$ Université de Lyon, Université Jean Monnet de Saint-Etienne, CNRS UMR5516 Laboratoire Hubert Curien, \\ F-42000 Saint-Etienne, France. \\ ' Institut d'Optique - Graduate School, 16 rue du professeur Benô̂t Lauras, 42000 Saint-Etienne, France. \\ ${ }^{\mathrm{c}}$ Ecole Polytechnique Fédérale de Lausanne (EPFL), School of Computer and Communication Sciences, \\ CH-1015 Lausanne, Switzerland.
}

\begin{abstract}
Reliable color reproduction can be achieved by establishing, using a model, the correspondence between the spectral reflectance of the printed surface and the amounts of deposited inks. In this paper, we review the main models proposed in the literature, recall how each one is calibrated and compare their prediction accuracy for several print sets. The presented models are the Yule-Nielsen modified spectral Neugebauer model, its cellular instance, the Clapper-Yule model, its instance supporting low scattering, and the two-by-two halftone independent dot centering model. The Yule-Nielsen and Clapper-Yule based models are combined with ink spreading assessment methods that account for the interaction between light, inks and paper. The prediction accuracy of the different models has been tested for several sets of printed colors on different supports, with different printing systems, different inks and different halftoning methods. These results show how the predictive performances and the model parameters vary according to the different printing setups.
\end{abstract}

\section{Introduction}

Following the rapid expansion of digital imaging and digital printing, color management of printed color images is a challenge due to the very large variety of media [1]. These media comprise different types of printing support (paper, plastic...), different types of inks (dye based, pigment based, etc.), different printing technology (e.g. offset lithography, inkjet, electrophotography, etc.) as well as halftoning techniques (mutually rotated clustered dots, error diffusion...) which have a large influence on the rendering of a same printed color image. The most promising method to calibrate printing systems for a given set of

\footnotetext{
${ }^{1}$ Parts of the present paper will appear in the Handbook of Digital Imaging, Chapter "Base models for color halftone reproduction", Ed. Michael Kriss, to be be published by J. Wiley.
} 
consumables and printing parameters is to use a spectral prediction model calibrated from a limited set of test samples.

The first color prediction models were proposed in the first part of the $20^{\text {th }}$ century. Neugebauer [2] made in 1933 a first attempt to predict the CIEXYZ tristimulus values of halftone colors. This approach was then improved by Yule and Nielsen in the early 1950s [3] in order to account for non-linear phenomena due to scattering of light by the printing support, and by Viggiano [4] in the 1990s who showed that a model based on spectral reflectances was more accurate than a model based on CIE-XYZ tristimulus values. Balasubramanian [5] showed that the model can be improved by accounting for the physical dot gain of each ink. More recently, Hersch et al. [6] proposed a decisive improvement with a method to estimate the amount of spreading of the different inks on the surface. With this last improvement, accurate predictions can be achieved for the spectral reflectance of many kinds of surfaces printed with classical halftoning techniques. In case of halftoning with custom inks [7], accurate predictions are also possible thanks to the two-by-two dot centering model proposed by Wang [8]. All these models are based on a combination of the spectral reflectances of the solid tones, i.e. color patches where each ink is either printed at full surface coverage or not printed. In order to increase the prediction accuracy of these models, Heuberger et al. [9] introduced the idea of combining the spectral reflectance of more tones, i.e. the solid tones and specific halftones (e.g. the halftones where each ink is printed at the surface coverage $0,0.5$ or 1). Balasubramanian transposed this idea to the Yule-Nielsen spectral Neugebauer model, thus creating a "Cellular Yule-Nielsen model" [5].

In 1953, Clapper and Yule proposed a different approach by modeling the interaction between light and the print halftone [10]. This model, combined with the method of Hersch et al. to estimate the amount of ink spreading, also provides good prediction, especially for high halftone screen frequencies. In the same way as for the previous models, special tones are printed and their spectral reflectances are measured to calibrate the model. Since the reflections and refractions of rays at the print-air interface are explicitly taken into account, the illumination and observation geometries selected for the measurements are expressed as model parameters in the equations.

Various reviews of predictive accuracy of models were proposed in the past. In 1993, Rolleston and Balasubramanian compared the accuracy of various types of Neugebauer models [11], including the Spectral Neugebauer, the Yule-Nielsen modified spectral Neugebauer and the cellular Yule-Nielsen modified spectral Neugebauer model, with different levels of cellular subdivisions. In 2000, Wyble and Berns did a thorough review of these various types of Neugebauer models [12] also including a brief description of extended Murray-Davies models [13] where the paper and ink reflectances are considered to be functions of the ink surface coverages. In the present contribution, we review the prediction models that offer according to our experience the best prediction accuracy for various print 
technologies. We therefore focus on the ink spreading enhanced Yule-Nielsen spectral Neugebauer and Clapper-Yule models, as well as on the two-by-two dot centering model which offers to some extent halftone independent color predictions.

We first recall basic definitions relative to halftones (Section 2) and present the predictive equations of different models (Section 3). We then explain how the models are calibrated, especially in order to assess the spreading of the inks (Section 4), and how predictions are made for a given halftone color (Section 5). In Section 6, we show how to adapt the presented three ink halftone models to four-ink halftones. Section 7 is dedicated to the two-by-two dot centering model, which is noticeably different from the other models. The comparison of the prediction accuracies of the different models is presented in Section 8 by tests performed with different papers, different ink sets, different printing systems, and different measurement geometries.

\section{Inks and colorants in halftones}

A halftone color is obtained by depositing each ink according to a specific binary pattern whose surface coverage determines the amount of ink per unit area and therefore the spectral absorbance of the print surface. Generally, the hafltone patterns associated to the different inks partially overlap each other. They create on the surface a mosaic of areas with different colors. The number of these elementary colors, hereinafter denoted "colorants" but also often called "Neugebauer primaries", is limited and depends only on the number of inks. In $k$-ink printing, the presence or absence of each ink yields $2^{k}$ possible combinations, therefore $2^{k}$ colorants. For example, classical CMY halftones contain $2^{3}=8$ colorants: white (no cyan, no magenta, no yellow), cyan (cyan, no magenta, no yellow), magenta (no cyan, magenta, no yellow), yellow (no cyan, no magenta, yellow), red (no cyan, magenta, yellow), green (cyan, no magenta, yellow), blue (cyan, magenta, no yellow) and black (cyan, magenta and yellow).

In classical clustered-dot or error diffusion prints, the halftone screens of the different inks are statistically independent. Thus, the fractional area occupied by each colorant can be deduced from the surface coverages of the inks according to Demichel's equations [14]. For cyan, magenta and yellow inks with respective surface coverages $c, m$, and $y$, the surface coverages of the eight colorants are: 


$$
\begin{aligned}
& a_{w}=(1-c)(1-m)(1-y) \quad: \text { white } \\
& a_{c}=c(1-m)(1-y) \quad: \text { cyan } \\
& a_{m}=(1-c) m(1-y) \quad \text { : magenta } \\
& a_{y}=(1-c)(1-m) y \quad: \text { yellow } \\
& a_{r}=(1-c) m y \quad \text { : red } \\
& a_{g}=c(1-m) y \quad \text { :green } \\
& a_{b}=c m(1-y) \quad \text { : blue } \\
& a_{k}=\text { cmy } \quad \text { : chromatic black }
\end{aligned}
$$

Color patches where all the inks have a surface coverage 0 or 1 contain one colorant covering uniformly the whole patch area. They are called "solid colorant patches" and their spectral reflectances, denoted $R_{j}(\lambda)$ for colorant $j$, can be easily measured using a spectrophotometer. These measured spectral reflectances embody physical and optical phenomena related to the interaction between light, ink halftones and paper. Solid colorant reflectances are combined in order to predict the spectral reflectance of printed halftone colors.

\section{Predictive equations}

Each model relies on an equation that models the light-print interaction. Let us imagine a flux of photons striking the printed surface: some photons are directly reflected by the print-air interface; the other ones penetrate the print through the different colorant areas, travel within the printing support where they may be scattered multiple times and then come back to the surface. At the surface, some of the photons exit the print and the other photons are internally reflected by the print-air interface and travel once again into the printing support, where they are scattered and reflected. Due to the multiple reflection cycles, photons will traverse the different colorant areas. Finally, the detector collects the non-absorbed photons exiting the print. Note that the initial orientation of the photon (illumination geometry) and the position of the detector (observation geometry) may have an influence on the measured spectral reflectance.

As a consequence of the complex paths followed by the photons within the print, the attenuations contributed by the colorants to the final spectral reflectance of a halftone are generally non-linear. They are modeled by an equation where each printed solid colorant $j$ is characterized by its individual spectral reflectance $R_{j}(\lambda)$. This reflectance is measured on a patch with the solid colorant printed alone on paper. The surface coverages of the colorants forming the halftone are a priori unknown because at printing time the ink dots may spread. How to fit them by carrying out measurements of specific halftones is explained in Section 5 . In the present section, we focus on the different equations proposed in the literature expressing the spectral reflectances of a halftone as a function of the reflectances of the individual solid colorants. 
Spectral Neugebauer model. The simplest model considers that the contribution of each colorant $i$ to the spectral reflectance $R(\lambda)$ of a halftone color is proportional to its su iface coverage $a_{j}$ :

$$
R(\lambda)=\sum_{j=1}^{2^{k}} a_{j} R_{j}(\lambda),
$$

where $k$ denotes the number of primary inks and $R_{j}(\lambda)$ is the individual spectral reflectance of colorant $i$ printed alone on the surface. This model is accurate only if the travel of light into the print is short, i.e. if the majority of photons traverse at most one colorant along their path across the print. This is the case for halftone colors printed on specular reflectors (mirrors, nonscattering films) or if the halftone ink dots are very large, thus limiting the chance for photons to transit from one colorant to another one even though they are scattered by the printing support. These conditions, however, are satisfied only in special cases. Most of the time, photon paths traverse different colorant areas and the linear equation (2) does not yield accurate predictions.

Yule-Nielsen modified spectral Neugebauer model. By studying the non-linear relationship between the reflectance of halftones and the reflectance of the individual solid colorants, Yule and Nielsen established an empirical law that Viggiano used as a correction of the Neugebauer equation $[3,4,15]$. The resulting Yule-Nielsen modified spectral Neugebauer equation is the following:

$$
R(\lambda)=\left[\sum_{j=1}^{2^{k}} a_{j} R_{j}^{1 / n}(\lambda)\right]^{n},
$$

where $n$ is a scalar parameter related to the proportion of photon paths going through different colorant areas. It depends on the printing support, the halftone screen frequency and the absorbance of the inks. The Neugebauer equation is a special case of equation (3) when $n=1$. The $n$ value increases when the halftone screen frequency increases, or equivalently, the size of the ink dots decreases, or when the printing support is more scattering. It is experimentally determined when calibrating the model.

Since the spectral reflectances of the colorants have been measured at certain illumination and observation geometries, the spectral reflectances of halftones predicted by equation (3) are valid for the same illumination and observation geometries. The equation cannot account for a change of illumination or observation geometry.

Cellular Yule-Nielsen model. In order to provide higher prediction accuracy, Heuberger et al. [9] proposed to divide the full domain of the ink surface variations into sub-domains. The vertices of these subdomains, corresponding to specific nominal surface coverages of the inks, have known measured reflectances. Since the subdomains span a smaller subset of the color 
space compared with the original domain, the spectral prediction model relying on functions of subdomain vertex reflectances provides more accurate predictions. Subdomains may be created by dividing the CMY surface coverage unit cube into 8 subcubes (subdomains), formed by combinations of $0 \%, 50 \%$ and $100 \%$ nominal surface coverages of the cyan, magenta and yellow inks (Figure 1). With such a subdivision, the number of primary reflectances increases from 8 to 27. Each subdomain, for example the one formed by ink coverages varying between $0 \%$ and $50 \%$, forms itself a spectral Neugebauer model formed by 8 of the 27 primary reflectances. Balasubramanian [5] has shown that the cellular subdivision is applicable to the Yule-Nielsen spectral Neugebauer model. In order to achieve even higher prediction accuracy, one may further subdivide each subcube into subsubcubes. However, for 3 inks, this additional subdivision step would increase the number of measurements to $5^{3}=125$ and for 4 inks to $5^{4}=625$.

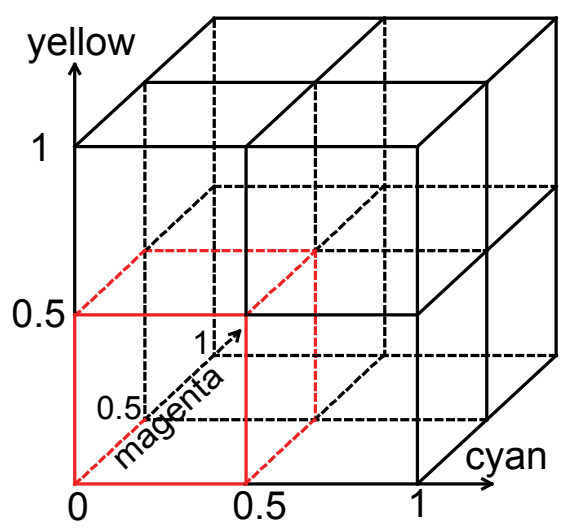

Figure 1. Subdivision of the ink surface coverage space into subdomains produced by all combinations of 0, 0.5 and 1 surface coverages of the three inks.

Let us describe in detail the cellular Yule-Nielsen model with one subdivision level [16]. The subdomain primaries are formed by surface coverages at all combinations of $0 \%, 50 \%$ and $100 \%$ surface coverages $\left(3^{3}=27\right.$ combinations $)$. Figure 2 illustrates a subdomain where the cyan, magenta and yellow ink surface coverages vary from 0 to 0.5 . Within each subdomain, the surface coverages are normalized. For an arbitrary cellular subdivision and with cyan, magenta and yellow ink surface coverages $c, m, y$ within a subdomain delimited by $c^{\prime} \in\left[c_{l}, c_{h}\right], m^{\prime} \in\left[m_{l}, m_{h}\right]$, and $y^{\prime} \in\left[y_{l}, y_{h}\right]$, the normalized $c^{\prime}, m^{\prime}, y^{\prime}$ ink coverages are

$$
c^{\prime}=\frac{c-c_{l}}{c_{h}-c_{l}} ; m^{\prime}=\frac{m-m_{l}}{m_{h}-m_{l}} ; y^{\prime}=\frac{y-y_{l}}{y_{h}-y_{l}}
$$




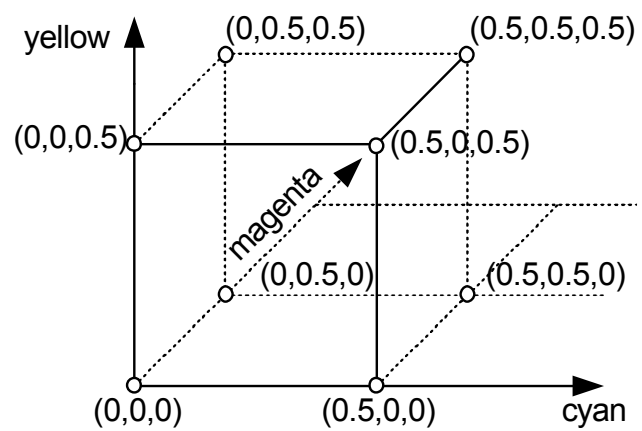

Figure 2. The subdomain with nominal surface coverages between $0 \%$ and $50 \%$. At the vertices of the subdomain cube, subdomain primary reflectances $R_{c, m, y}(\lambda)$ have been measured.

The predicted reflectance $R(\lambda)$ of a halftone of surface coverages $c \in\left[c_{l}, c_{h}\right], m \in\left[m_{l}, m_{h}\right]$, $y \in\left[y_{l}, y_{h}\right]$ is obtained by tri-linear interpolation of cube vertex reflectances:

$$
\begin{aligned}
& R(\lambda)=\left[\left(1-c^{\prime}\right)\left(1-m^{\prime}\right)\left(1-y^{\prime}\right) R_{c l, m l, y l}(\lambda)^{1 / n}\right. \\
& +c^{\prime}\left(1-m^{\prime}\right)\left(1-y^{\prime}\right) R_{c h, m l, y l}(\lambda)^{1 / n} \\
& +\left(1-c^{\prime}\right) m^{\prime}\left(1-y^{\prime}\right) R_{c l, m h, y l}(\lambda)^{1 / n} \\
& +\left(1-c^{\prime}\right)\left(1-m^{\prime}\right) y^{\prime} R_{c l, m l, y h}(\lambda)^{1 / n} \\
& +\left(1-c^{\prime}\right) m^{\prime} y^{\prime} R_{c l, m h, y h}(\lambda)^{1 / n} \\
& +c^{\prime}\left(1-m^{\prime}\right) y^{\prime} R_{c h, m l, y h}(\lambda)^{1 / n} \\
& +c^{\prime} m^{\prime}\left(1-y^{\prime}\right) R_{c h, m h, y l}(\lambda)^{1 / n} \\
& \left.+c^{\prime} m^{\prime} y^{\prime} R_{c h, m h, y h}(\lambda)^{1 / n}\right]^{n}
\end{aligned}
$$

where $R_{c x, m x, y x}(\lambda)$ represents the measured spectral reflectance at surface coverages $(c, m, y)$ of the cyan, magenta and yellow inks, either at the low end of the interval $(x=l)$ or at the high end of the interval $(x=h)$. For accurate prediction accuracy, the normalized surface coverages $c^{\prime}, m^{\prime}, y^{\prime}$ should be replaced by effective normalized surface coverages $c_{\text {eff }}, m_{\text {eff }}$, $y_{\text {eff' }}$ fitted simultaneously by minimizing the difference between predicted and measured reflectance of the halftone located at the center of the corresponding subcube (see Section 5).

Clapper-Yule model. In contrast to the previous models, the Clapper-Yule model is based on the physical description of the light paths in the print. The printing support is characterized by its intrinsic spectral reflectance $r_{g}(\lambda)$. The colorants are characterized by their spectral transmittance, denoted $t_{j}(\lambda)$. The printed support and the inks are assumed to have the same index of refraction, generally 1.5, which determines the Fresnel reflections and transmission at the print-air interface. The Clapper-Yule equation is: 


$$
R(\lambda)=r_{s}+\frac{T_{\text {in }} T_{\text {out }} r_{g}(\lambda)\left[\sum_{j=1}^{2^{k}} a_{j} t_{j}(\lambda)\right]^{2}}{1-r_{i} r_{g}(\lambda)\left[\sum_{j=1}^{2^{k}} a_{j} t_{j}^{2}(\lambda)\right]}
$$

where $r_{s}, r_{i}, T_{i n}$ and $T_{\text {out }}$ are constant parameters characterizing the print-air interface depending on the index of refraction of the print and on the measuring geometry, but not on wavelength. Table 1 gives numerical values for these terms when the refractive index is 1.5 , for three typical measuring geometries: (i) diffuse incident light, reflectance captured at $8^{\circ}$ with specular reflection included (di: $8^{\circ}$ ), (ii) diffuse incident light, reflectance captured at $8^{\circ}$ with specular reflection excluded (de: $8^{\circ}$ ), and (iii) collimated incident light at $45^{\circ}$, reflectance captured at $0^{\circ}\left(45^{\circ}: 0^{\circ}\right)$.

Table 1. Parameters of the Clapper-Yule model for a refractive index of 1.5 and for three typical measuring geometries

\begin{tabular}{ccccc} 
Measuring geometry & $r_{s}$ & $T_{\text {in }}$ & $T_{\text {out }}$ & $r_{i}$ \\
\hline $\operatorname{di}: 8^{\circ}$ & 0.04 & 0.91 & 0.43 & 0.60 \\
$\operatorname{de}: 8^{\circ}$ & 0 & 0.91 & 0.43 & 0.60 \\
$45^{\circ}: 0^{\circ}$ & 0 & 0.95 & 0.43 & 0.60 \\
\hline
\end{tabular}

In the case of a solid colorant patch, where one colorant covers the whole surface, the Clapper-Yule equation (6) becomes

$$
R(\lambda)=r_{s}+\frac{T_{\text {in }} T_{\text {out }} r_{g}(\lambda) t_{j}^{2}(\lambda)}{1-r_{i} r_{g}(\lambda) t_{j}^{2}(\lambda)}
$$

The spectral parameters of the model, $r_{g}(\lambda)$ and $t_{j}(\lambda)$, cannot be measured directly. They are deduced from the spectral reflectance of the solid colorant patches [17]. The intrinsic spectral reflectance $r_{g}(\lambda)$ of the paper substrate is deduced from the measured spectral reflectance $R_{w}(\lambda)$ of the unprinted paper ("white" colorant patch):

$$
r_{g}(\lambda)=\frac{R_{w}(\lambda)-r_{s}}{T_{\text {in }} T_{\text {out }}+r_{i}\left(R_{w}(\lambda)-r_{s}\right)}
$$

The spectral transmittances of colorants are deduced from the measured spectral reflectances of the other solid colorants patches, $R_{j}(\lambda)$ :

$$
t_{j}(\lambda)=\sqrt{\frac{1}{r_{g}(\lambda)} \cdot \frac{R_{j}(\lambda)-r_{s}}{T_{\text {in }} T_{\text {out }}+r_{i}\left(R_{j}(\lambda)-r_{s}\right)}}
$$

The Clapper-Yule equation is based on a description of the multiple reflections of light between the diffusing substrate and the print surface through the ink layers. It assumes that light can travel a long distance within the paper substrate, thus traversing a different colorant 
at each internal reflection in the multiple reflection process, independently of the colorant from which light entered the print. It is therefore adapted to high halftone screen frequencies.

Low scattering Clapper-Yule model. In order to support low and intermediate screen frequencies, Hersch et al. [18] proposed a linear weighting between the Clapper-Yule model adapted to high screen frequencies and the Spectral Neugebauer model adapted to low screen frequencies. This yields the following equation which will be referred to as the low scattering Clapper-Yule model:

$$
R(\lambda)=b R_{S N}(\lambda)+(1-b) R_{C Y}(\lambda)
$$

where $b$ is a parameter which indicates how close the model is to the Spectral Neugebauer model. $R_{S N}(\lambda)$ is given by Eq. (2), where $R_{j}(\lambda)$ is the measured colorant reflectance from which according to formula (8) and (9) both the intrinsic paper reflectance $r_{g}(\lambda)$ and the spectral transmittances $t_{j}(\lambda)$ of the colorants may be deduced. $R_{C Y}(\lambda)$ is the Clapper-Yule equation (6). The developed version of Eq. (10) is

$$
R(\lambda)=r_{s}+T_{\text {in }} T_{\text {out }}\left[b\left(\sum_{j=1}^{2^{k}} a_{j} \frac{r_{g}(\lambda) t_{j}^{2}(\lambda)}{1-r_{i} r_{g}(\lambda) t_{j}^{2}(\lambda)}\right)+(1-b) \frac{\left(\sum_{j=1}^{2^{k}} a_{j} t_{j}(\lambda)\right)^{2} r_{g}(\lambda)}{1-r_{i} r_{g}(\lambda)\left(\sum_{j=1}^{2^{k}} a_{j} t_{j}^{2}(\lambda)\right)}\right]
$$

\section{Calibration of the Yule-Nielsen and Clapper Yule models}

In order to account for the specific optical properties of the used media (paper, inks) and printing setup (printer, halftoning technique), the models need to be calibrated from spectral measurement of a small set of printed samples. This set of sample comprises the $2^{k}$ solid colorant patches, where $k$ is the number of primary inks. In order to fit the amount of spreading for each ink, the set of calibration samples also comprises patches where a single ink is halftoned. 


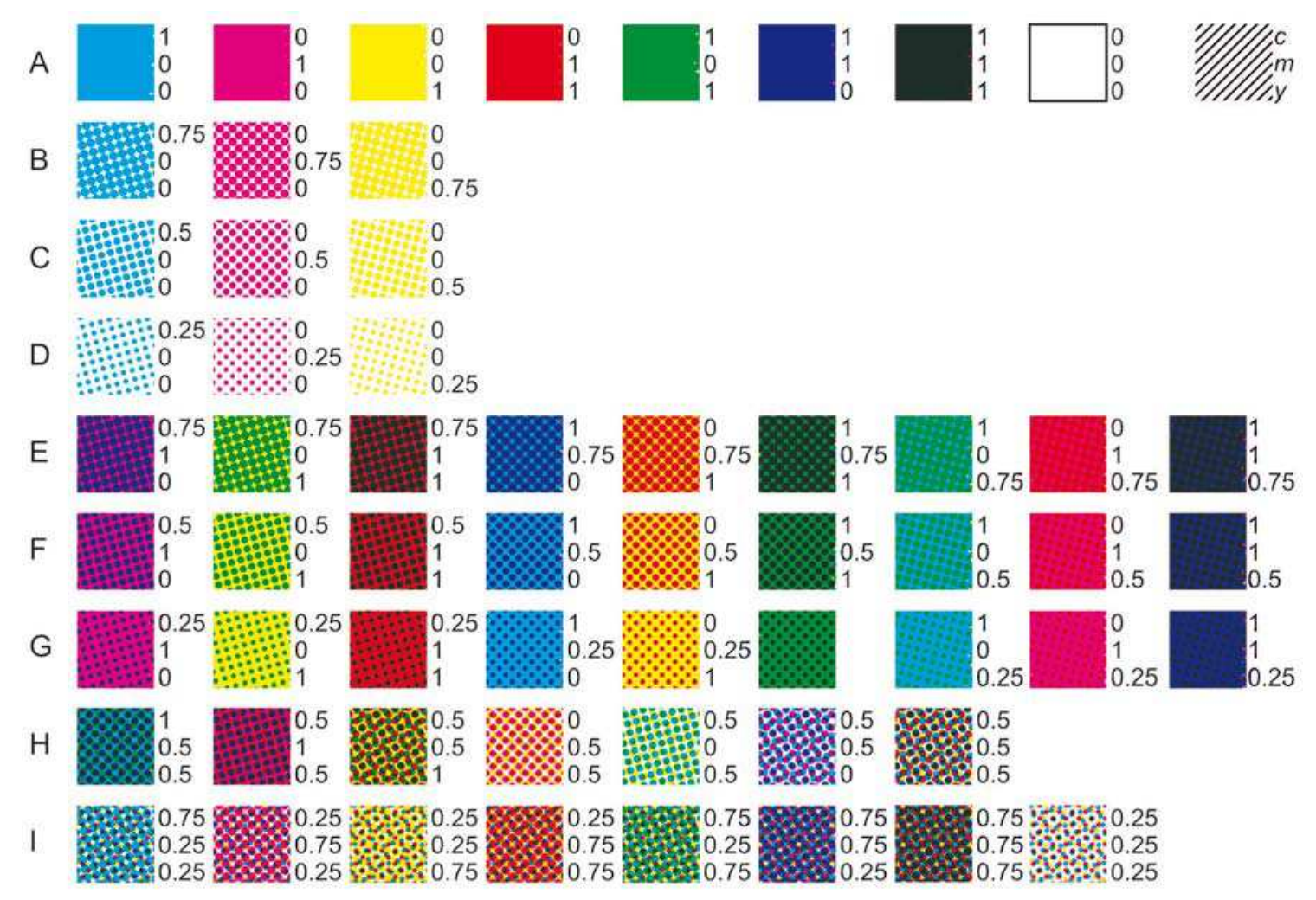

Figure 3. Color patches used for the calibration of the different models in the case of CMY halftones. The numbers at the right of each patch give the surface coverages of respectively cyan, magenta and yellow inks.

Figure 3 represents a typical set of patches used for the calibration of CMY spectral prediction models. Row A contains the eight solid colorant patches; rows B, C and D contain the halftones where one ink $i$ is printed alone on paper at the nominal surface coverages $q_{i}=$ $0.25,0.5$ or 0.75 ; rows $\mathrm{E}, \mathrm{F}$ and $\mathrm{G}$ contain the halftones where one ink $i$ is printed at the nominal surface coverages $q_{i}=0.25,0.5$ or 0.75 and at least one of the other inks at the surface coverage 1 . Row $\mathrm{H}$ contain the halftones where two inks are printed at the norminal surface coverage 0.5 and the third ink at the surface coverage 1 or 0 , and row I contains the halftones where each of the three inks are printed at a surface coverage 0.25 or 0.75 .

Basic Ink Spreading assessment method. The basic calibration of ink spreading relies, for three inks, on the 9 patches represented in the rows B, C and D. These halftones, whose spectral reflectance is denoted $R_{q_{i}}^{(m)}(\lambda)$, contain two colorants: the ink which should occupy a fractional area $q_{i}$ and the paper white which should occupy the fractional area $1-q_{i}$. Applying the model's equation with these two colorants and these surface coverages should predict a spectral reflectance equal to the measured one. However, due to the fact that the effective ink surface coverage is different from the nominal one, these two reflectances are not the same. One thus fits the effective surface coverage $q_{i}^{\prime}$ as the $Q_{i}$ which minimizes the deviation between predicted spectrum $R\left(Q_{i}, \lambda\right)$ and measured spectrum $R_{q_{i}}^{(m)}(\lambda)$, by 
quantifying the deviation either by the sum of square differences of the components of the two spectra, i.e.

$$
q_{i}^{\prime}=\underset{0 \leq Q_{i} \leq 1}{\arg \min } \sum_{\lambda=380 \mathrm{~nm}}^{730 \mathrm{~nm}}\left[R\left(Q_{i}, \lambda\right)-R_{q_{i}}^{(m)}(\lambda)\right]^{2}
$$

or by the sum of square difference of the components of their logarithm, i.e.

$$
q_{i}^{\prime}=\underset{0 \leq Q_{i} \leq 1}{\arg \min } \sum_{\lambda=380 \mathrm{~nm}}^{730 \mathrm{~nm}}\left[\log R\left(Q_{i}, \lambda\right)-\log R_{q_{i}}^{(m)}(\lambda)\right]^{2}
$$

or by the corresponding color difference given e.g. by the CIELAB $\Delta E_{94}$ metric

$$
q_{i}^{\prime}=\underset{0 \leq Q_{i} \leq 1}{\arg \min } \Delta E_{94}\left(R\left(Q_{i}, \lambda\right), R_{q_{i}}^{(m)}(\lambda)\right)
$$

Eq. (12) is the most classical way of determining the effective surface coverage. Since the human visual system is more sensitive to differences at low intensity, the log of the spectra in Eq. (13) provides a higher weight to lower reflectance values. Fitting $q_{i}^{\prime}$ from the color difference metric sometimes improves the prediction accuracy of the model but complicates the optimization. Even at the optimal surface coverage $q_{i}^{\prime}$, the difference between the two spectra is rarely zero and provides a first indication of the prediction accuracy achievable by the model for the corresponding print setup.

Once the 9 effective surface coverages are computed, assuming that the effective surface coverage is 0 , respectively 1 , when the nominal surface coverage is 0 (no ink), respectively 1 (full coverage), we obtain three sets of $q_{i}^{\prime}$ values which, by linear interpolation, yield the continuous ink spreading functions $f_{i}$ (Figure 4).
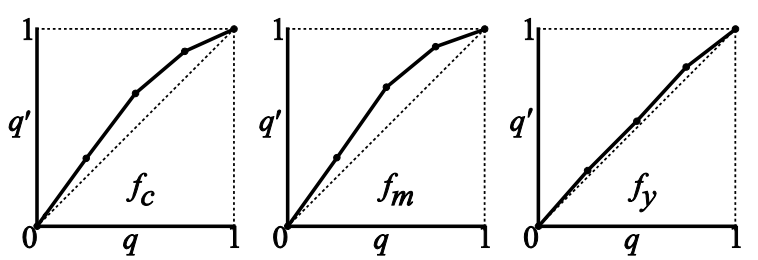

Figure 4. Example of ink spreading curves $f_{i}$ obtained by linear interpolation of the effective surface coverages $q_{i}^{\prime}$ which are deduced from measurement of patches with single-ink halftones (ink i) printed at nominal surface coverages $0.25,0.5$ and 0.75 .

A good approximation of the ink spreading functions $f_{i}$ may also be performed by quadratic interpolation between the points $(0,0),\left(0.5, q^{\prime}(0.5)\right)$ and $(1,1)$, i.e. by considering the parabola passing through these three points:

$$
f_{i}(q)=\left[2-4 q_{i}^{\prime}(0.5)\right] q^{2}+\left[4 q_{i}^{\prime}(0.5)-1\right] q
$$


where $q^{\prime}(0.5)$ denotes the effective surface coverage at the nominal surface coverage of 0.5 . For this approximation of the ink spreading fucntion, only one measurement per ink spreading curve is needed. For three inks, only the three patches represented in row $\mathrm{C}$ of Figure 3 are needed.

In the case of the Cellular Yule-Nielsen model, the ink spreading assessment method described above is performed in each of the eight subdomains represented in Figure 1. We thus consider for its calibration the 27 spectral reflectances of cellular Neugebauer primaries in which the surface coverage of each ink is $0,0.5$ or 1 (rows A, C, F and H in Figure 3). The ink spreading functions are calibrated from the spectral reflectances of eight halftones in which the surface coverage of the ink halftones are 0.25 and 0.75 (row I in Figure 3). These eight halftones are the centers of the subdomains of the ink surface coverage space. Each of the eight corresponding spectral reflectances enables fitting, in one optimization operation, the three effective surface coverages of respectively the cyan, magenta and yellow inks, which are then converted by quadratic interpolation into ink spreading functions having a parabolic shape. We thus obtain three ink spreading functions per subdomain, yielding a total of 24 ink spreading functions.

Superposition-dependent ink spreading assessment method. The basic ink spreading assessment method can be improved by observing that the amount of ink spreading depends on whether the ink is alone on the support or superposed with other inks. The superpositiondependent ink spreading method, for three inks, relies on the patches represented in the rows $\mathrm{B}$ to $\mathrm{G}$ of Figure 3. In rows $\mathrm{E}, \mathrm{F}$ and $\mathrm{G}$, the patches comprise one ink halftone superposed with either one or two other solid inks (surface coverage 1). The effective surface coverages are deduced from their spectral reflectance in the same way as in the basic ink spreading method by considering for each halftone the appropriate two colorants. One obtains 12 sets of effective surface coverages and therefore 12 ink spreading curves $q^{\prime}=f_{i / J}(q)$, where subscript $i / J$ denotes ink hafltone $i$ superposed with solid colorant $J$ (see Figure 5). As previously mentioned, parabolic interpolation enables reducing the number of calibration patches. By printing and measuring halftones at the nominal surface coverage of 0.5 , only the 12 patches shown in rows $\mathrm{C}$ and $\mathrm{F}$ of Figure 3 are needed for establishing the ink spreading curves. 

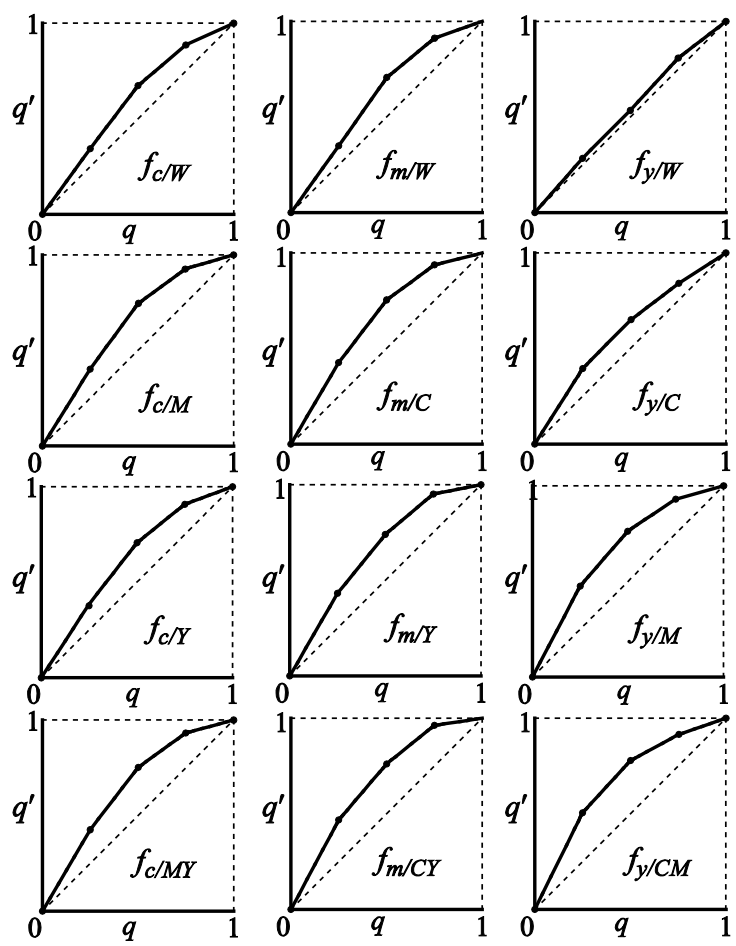

Figure 5. Example of ink spreading curves $f_{i / J}$ obtained by linear interpolation of the effective surface coverages $q_{i / J}^{\prime}$ which are deduced from measurement of patches with single-ink halftones (ink i) printed at nominal surface coverages $0.25,0.5$, and 0.75 and superposed with a solid layer of colorant $J$.

Experience has shown that compared with basic ink spreading, superposition-dependent ink spreading enables improving the prediction accuracy of the Yule-Nielsen and ClapperYule models by more than 50\% [6]. However, for the Cellular Yule-Nielsen model, superposition dependent ink spreading does not bring much improvement, since, within a subdomain, the ink spreading curves do not depend much on the superposition condition.

Independently of the selected prediction model and ink spreading calibration method, the highest prediction accuracy is achieved for colors printed and observed in the same conditions as the calibration patches: same support, same inks, same printing system, same halftoning method, same illumination conditions and same viewing direction. In addition, one should measure with the same geometry. If one of these requirements is not fulfilled, bad predictions may be obtained and the whole calibration procedure should be repeated with the modified configuration. Since the spectral parameters of the models and the ink spreading curves account for the many complex chemical, mechanical and optical phenomena underlying the light-paper-ink interaction, a calibration performed with a given printing support cannot be easily reused for prediction with a different printing support. The development of a simplified calibration procedure when either the support or the inks are replaced by similar ones is a major challenge for research in the coming years. The two-by-two dot centering model (presented in Section 6) represents an important progress. Despite requiring a calibration 
procedure based on a quite large number of printed patches [19], it can make valid spectral predictions for different halftoning methods. However, it makes the assumption that light entering the print does not propagate significantly beyond a one pixel sized neighbourhood or that neighbour colors evolve smoothly.

\section{Prediction}

Once the spectral parameters and the ink spreading functions are computed, the model is calibrated. One can predict the spectral reflectance of halftones having any nominal ink surface coverages $c_{0}, m_{0}$, and $y_{0}$. With the basic ink spreading method, the ink spreading functions $f_{i}$ directly provide the effective surface coverages $c, m$, and $y$ of the three inks:

$$
\begin{aligned}
c & =f_{c}\left(c_{0}\right) \\
m & =f_{m}\left(m_{0}\right) \\
y & =f_{y}\left(y_{0}\right)
\end{aligned}
$$

These effective ink surface coverages are plugged into the Demichel equations (1), which provide the effective surface coverages of the eight colorants. The general equation of the selected model finally predicts the spectral reflectance of the considered halftone. Predictions with the Cellular Yule-Nielsen model are carried out in a similar manner, but the basic ink spreading functions (16) are specific for each sub-domain.

When using the superposition-dependent ink spreading method, the nominal ink surface coverages $c_{0}, m_{0}$, and $y_{0}$ are converted into effective ink surface coverages $c, m$ and $y$ by accounting for the superposition-dependent ink spreading. The effective surface coverage of each ink is obtained by a weighted average of the ink spreading curves. The weights are expressed by the surface coverages of the respective colorants on which the ink halftone is superposed. For example, the weight of the ink spreading function $f_{c}$ (cyan halftone over the unprinted white substrate) is proportional to the surface of the underlying white substrate colorant, i.e. $(1-m)(1-y)$. In the case of three halftoned inks, effective surface coverages are obtained by performing a few iterations with Eq. (17):

$$
\begin{aligned}
& c=(1-m)(1-y) f_{c}\left(c_{0}\right)+m(1-y) f_{c / M}\left(c_{0}\right)+(1-m) y f_{c / Y}\left(c_{0}\right)+m y f_{c / M Y}\left(c_{0}\right) \\
& m=(1-c)(1-y) f_{m}\left(m_{0}\right)+c(1-y) f_{m / C}\left(m_{0}\right)+(1-c) y f_{m / Y}\left(m_{0}\right)+c y f_{m / C Y}\left(m_{0}\right) \\
& y=(1-c)(1-m) f_{y}\left(y_{0}\right)+c(1-m) f_{y / C}\left(y_{0}\right)+(1-c) m f_{y / M}\left(y_{0}\right)+c m f_{y / C M}\left(y_{0}\right)
\end{aligned}
$$

For the first iteration, $c=c_{0}, m=m_{0}$ and $y=y_{0}$ are taken as initial values on the right side of the equations. The obtained values of $c, m$ and $y$ are then inserted again into the right side of the equations, which yields new values of $c, m, y$ and so on, until the values of $c, m, y$ stabilize. The effective surface coverages of the colorants are calculated by plugging the obtained values for $c, m$ and $y$ into the Demichel equations. The spectral reflectance of the 
considered halftone is finally provided by the general equation of the selected model. Figure 6 show an overview of the prediction procedure.

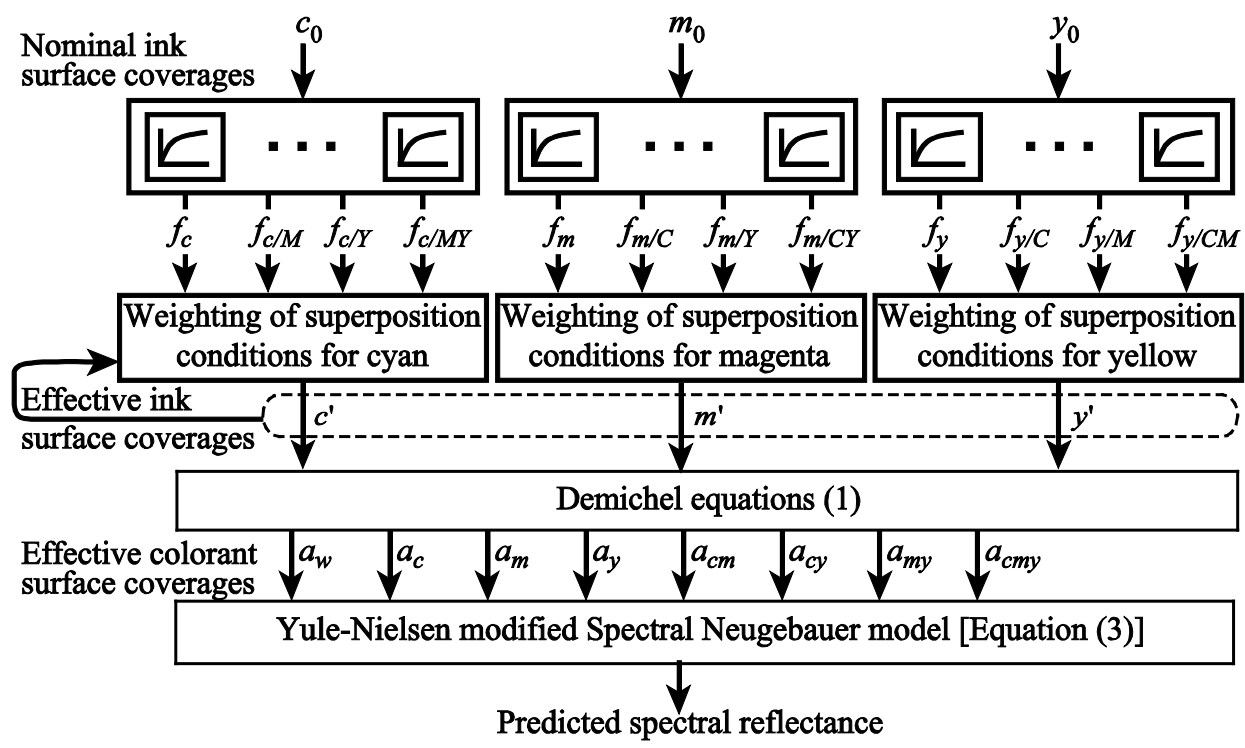

Figure 6. The ink spreading enhanced Yule-Nielsen modified Spectral

Neugebauer model with nominal ink surface coverages $\mathrm{c}_{0}, \mathrm{~m}_{0}, \mathrm{y}_{0}$; ink spreading curves $\mathrm{f}_{\mathrm{i} / \mathrm{JK}}$ of ink $\mathrm{i}$ superposed with solid inks $\mathrm{j}$ and $\mathrm{k}$; effective ink surface coverages $c^{\prime}, m^{\prime}, y^{\prime}$ and effective colorant surface coverages $a_{\mathrm{w}}$ to $a_{\mathrm{cmy}}$.

\section{Four-ink halftones}

The models presented above can be extended to four ink halftones in a straightforward manner, by increasing the number of spectral parameters and of inks spreading functions. The number of colorants becomes $2^{4}=16$ instead of $2^{3}=8$ for 3 -ink halftones. The corresponding 16 spectral parameters are colorant reflectances for the Yule-Nielsen modified Spectral Neugebauer model. For the Clapper-Yule model, they are the paper's intrinsic reflectance as well as 15 ink transmittances deduced from the reflectances of the 15 non-white colorants.

Regarding the ink spreading assessment method, the basic method relies on 4 ink spreading functions, and the superposition-dependent method relies on 32 ink spreading functions. In order to perform linear interpolation, each ink spreading function requires typically the measurement of three halftones $(0.25,0.5$ and 0.75 surface coverage). For quadratic interpolation, only one halftone at 0.5 surface coverage is generally sufficient.

At prediction time, once the effective ink surface coverages $u, v, w, x$ of the four inks are computed, the surface coverages of the 16 colorants are provided by the 4-ink Demichel equations: 


$$
\begin{aligned}
& a_{1}=(1-u) \cdot(1-v) \cdot(1-w) \cdot(1-x) \\
& a_{2}=u \cdot(1-v) \cdot(1-w) \cdot(1-x) \\
& a_{3}=(1-u) \cdot v \cdot(1-w) \cdot(1-x) \\
& a_{4}=(1-u) \cdot(1-v) \cdot w \cdot(1-x) \\
& a_{5}=(1-u) \cdot(1-v) \cdot(1-w) \cdot x \\
& a_{6}=u \cdot v \cdot(1-w) \cdot(1-x) \\
& a_{7}=u \cdot(1-v) \cdot w \cdot(1-x) \\
& a_{8}=u \cdot(1-v) \cdot(1-w) \cdot x \\
& a_{9}=(1-u) \cdot v \cdot w \cdot(1-x) \\
& a_{10}=(1-u) \cdot v \cdot(1-w) \cdot x \\
& a_{11}=(1-u) \cdot(1-v) \cdot w \cdot x \\
& a_{12}=u \cdot v \cdot w \cdot(1-x) \\
& a_{13}=u \cdot v \cdot(1-w) \cdot x \\
& a_{14}=u \cdot(1-v) \cdot v \cdot w \cdot x \\
& a_{15}=(1-u) \cdot v \cdot w \cdot x \\
& a_{16}=u \cdot v \cdot w \cdot x
\end{aligned}
$$

These effective colorant surface coverages are then used to weight the spectral reflectances or transmittances according to the model's general equation. The model equations for $k=4$ inks are similar to the equations for $k=3$ inks, see Eqs. (2), (3), (6), (11).

In the case where the fourth ink is black, the superposition of other ink halftones with black still gives black. But the superposition of a black ink halftone with another ink yields a clearly identifiable physical dot gain. Since there are 8 superposition conditions of a black halftone, the extension of Eq. (17) enabling computing effective surfaces coverages of black in case of CMYK prints comprises 8 terms:

$$
\begin{aligned}
k^{\prime} & =\left(1-c^{\prime}\right) \cdot\left(1-m^{\prime}\right) \cdot\left(1-y^{\prime}\right) \cdot f_{k}(k) \\
& +c^{\prime} \cdot\left(1-m^{\prime}\right) \cdot\left(1-y^{\prime}\right) \cdot f_{k / C}(k) \\
& +\left(1-c^{\prime}\right) \cdot m^{\prime} \cdot\left(1-y^{\prime}\right) \cdot f_{k / M}(k) \\
& +c^{\prime} \cdot m^{\prime} \cdot\left(1-y^{\prime}\right) \cdot f_{k / C M}(k) \\
& +\left(1-c^{\prime}\right) \cdot\left(1-m^{\prime}\right) \cdot y^{\prime} \cdot f_{k / Y}(k) \\
& +c^{\prime} \cdot\left(1-m^{\prime}\right) \cdot y^{\prime} \cdot f_{k / C Y}(k) \\
& +\left(1-c^{\prime}\right) \cdot m^{\prime} \cdot y^{\prime} \cdot f_{k / M Y}(k) \\
& +c^{\prime} \cdot m^{\prime} \cdot y^{\prime} \cdot f_{k / C M Y} \cdot(k)
\end{aligned}
$$

The Cellular Yule-Nielsen modified Spectral Neugebauer model relies on $2^{4}=16$ subdomains and $3^{4}=81$ colorants (combinations of inks printed each one at surface coverages $0,0.5$ or 1 ). In order to establish the $4 \times 16=64$ ink spreading functions, 16 halftones (one at each subdomain center) are needed. The ink spreading functions are obtained in the same way as for three inks, and the predictions are performed using the 4 ink Demichel equations (18). 
When operating CMYK prediction models in inverse mode, i.e. when fitting surfaces coverages of the inks so as to obtain a given reflectance, many solutions may arise, due to the fact that the superposition of approximately equal surface coverages of CMY ink halftones yield chromatic gray tones similar to the pure gray tones producible with black ink halftones. In order to distinguish between chromatic black and pure black halftones, one may extend the spectral measurements to the near infrared wavelength range, i.e. between $730 \mathrm{~nm}$ and 850 nm. Classical CMY dye-based inks do not absorb light in this range, whereas pigment-based black inks generally absorb light in the near infrared wavelength range [20].

\section{The two-by-two dot centering model}

Ideally, a single printed dot is a perfect square of uniform ink thickness. In practice it looks more like an oval with a non-uniform thickness profile. Its exact size and thickness profile depend whether its neighborhood pixel locations are printed or not. Wang [8] developed a method, where only a two-by-two pixel neighborhood is analyzed. The vertex of each square forming a printable pixel is the center of a two-by-two pixel neighborhood. An elementary square of the size of one pixel, called "two-by-two" square, is laid out across the considered 4 neighboring pixels (Figure 7).

(a)
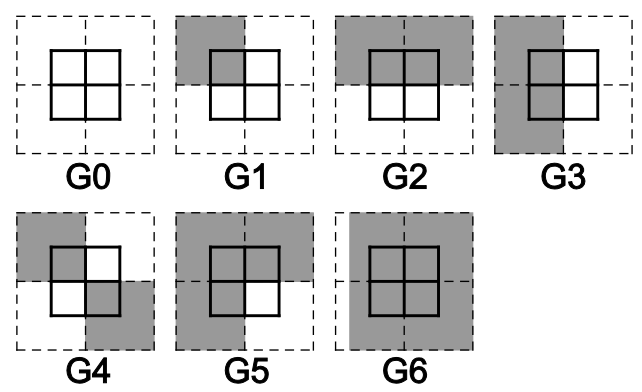

G3

(b)

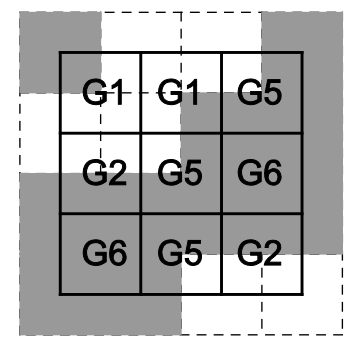

Figure 7. (a) Representative two-by-two square patterns $G 0$ to G6 for single ink prints and (b) example of a $4 \times 4$ pixel halftone, overlaid by its corresponding two-by-two square patterns.

Let us first consider a single ink print. Each two-by-two square is representative for the real printed layout at the junction of its 4 neighboring printable pixels. Since each pixel associated with a two-by-two square can be on or off, the two-by-two square can have $2^{4}=16$ different reflectance values. By taking into account horizontal, vertical and central symmetries, the 
number of representative reflectance values of single ink two-by-two squares that need to be differentiated is reduced to 7 (Figure 7). These 7 different reflectances can be learned by measuring the reflectances of tiles, each one composed of only one of these representative two-by-two patterns, repeated over a large surface. Figure 8 shows the calibration tiles associated with the corresponding representative two-by-two squares.

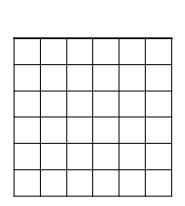

G0

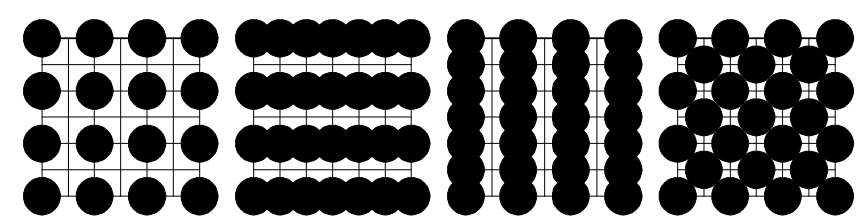

G2

G3

G4

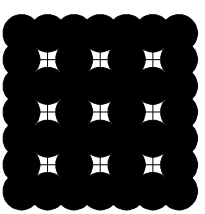

G5

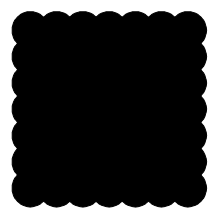

G6

Figure 8. Calibration tiles associated with the corresponding representative twoby-two squares.

In order to predict the reflectance of any input halftone or pattern image with the learned representative two-by-two patterns, one traverses the input image, by moving a two-by-two unit square from pixel square vertex to pixel square vertex, finding its associated representative pattern and creating an output bitmap formed of two-by-two unit squares labeled as G0 to G6 (Figure 7). The input image is now segmented into two-by-two labeled unit squares. Since the "microscopic" reflectance of each two-by-two labeled unit square is known, the "macroscopic" reflectance of a larger tile can be computed, for example a tile covering the area of a halftone. The reflectance of an image area formed by an assembly of two-by-two unit square reflectances may be calculated with the Yule-Nielsen modified spectral Neugebauer model (YNSN), according to Equation (20), where $i_{m}$ is the number of occurrences of representative two-by-two pattern $G_{m}$ in the considered area and $R_{m}(\lambda)$ is its corresponding measured spectral reflectance:

$$
R^{1 / n}(\lambda)=\frac{\sum_{m=0}^{6} i_{m} \cdot R_{m}^{1 / n}(\lambda)}{\sum_{m=0}^{6} i_{m}}
$$

The two-by-two centering model can be extended to multiple ink prints [21]. For dots within a two-by-two tile being printed with more than the black and white colors, the number of patterns that can occur within a two-by-two tile increases significantly. The number of possible arrangements for the four printed dots of a two-by-two tile and for $N$ solid colorants is $N^{4}$. In the case of a CMY print ( 8 colorants), there are $8^{4}=4096$ possible colors arrangements within a single two-by-two tile. If we remove both the horizontal and the vertical symmetries, $P(8)=1072$ independent patterns remain. In the case of 4 inks, there are 16 solid colorants and $16^{4}=65536$ possible arrangements. By removing horizontal and 
vertical symmetries, $P(16)=16576$ independent patterns remain. Let us rewrite Eq. (20) for the case of color predictions with $N$ solid colorants

$$
R^{1 / n}(\lambda)=\frac{\sum_{m=0}^{P(N)-1} i_{m} \cdot R_{m}{ }^{1 / n}(\lambda)}{\sum_{m=0}^{P(N)-1} i_{m}}
$$

For $N$ solid colorants, the number of patterns $P(N)$ remaining after considering horizontal and vertical symmetries can be calculated as follows. We first consider the total number of arrangement $Q(N)=N^{4}$. In the following arrangements, a horizontal or vertical symmetry does not induce two different patterns: (a) same color on all 4 pixels, (b) in respect to horizontal symmetry, same color on the two horizontal neighboring pixels and (c) in respect to vertical symmetry, same color on two neighboring vertical pixels. Table 2 gives the number of arrangements for these cases, both for 3-ink and 4-ink prints and shows how to compute the number of representative patterns.

Table 2. Calculation of symmetry reduced representative patterns, where $c_{1}$ represents one colorant and $c_{2}$ represents another colorant.

\begin{tabular}{|c|c|c|c|}
\hline & & 3 inks ( $N=8$ colorants) & 4 inks $(N=16$ colorants $)$ \\
\hline Uniform color of all 4 pixels & & 8 arrangements & 16 arrangements \\
\hline $\begin{array}{l}\text { Horizontal neighbors of } \\
\text { uniform color, but vertical } \\
\text { neighbors of different colors }\end{array}$ & $\begin{array}{ll}c_{1} & c_{1} \\
c_{2} & c_{2}\end{array}$ & $\begin{array}{l}\text { Select } 2 \text { from } 8 \text { colorants } \\
\left(\begin{array}{l}8 \\
2\end{array}\right)=28 \text { arrangements }\end{array}$ & $\begin{array}{l}\text { Select } 2 \text { from } 16 \text { colorants } \\
\left(\begin{array}{c}16 \\
2\end{array}\right)=120 \text { arrangements }\end{array}$ \\
\hline $\begin{array}{l}\text { Vertical neighbors of } \\
\text { uniform color, but horizontal } \\
\text { neighbors of different colors }\end{array}$ & $\begin{array}{ll}c_{1} & c_{2} \\
c_{1} & c_{2}\end{array}$ & $\begin{array}{l}\text { Select } 2 \text { from } 8 \text { colorants } \\
\left(\begin{array}{l}8 \\
2\end{array}\right)=28 \text { arrangements }\end{array}$ & $\begin{array}{l}\text { Select } 2 \text { from } 16 \text { colorants } \\
\left(\begin{array}{c}16 \\
2\end{array}\right)=120 \text { arrangements }\end{array}$ \\
\hline $\begin{array}{l}\text { Total number of non- } \\
\text { symmetric arrangements }\end{array}$ & $\begin{array}{ll}c_{1} & c_{2} \\
c_{1} & c_{2}\end{array}$ & $8+28+28=64$ & $16+120+120=256$ \\
\hline $\begin{array}{l}\text { Symmetry reduced number of } \\
\text { representative patterns } P(N)\end{array}$ & & $\frac{4096-64}{4}+64=1072$ & $\frac{65536-256}{4}+256=16576$ \\
\hline
\end{tabular}

One may significantly reduce the number of calibration tile measurements by predicting the reflectances of the large majority of representative two-by-two patterns. The predictions rely on a small subset of measured two-by-two pattern tiles [19], typically $10 \%$ of the total number of representative pattern tiles.

The present two-by-two spectral prediction method is to some extent halftone independent. After measurement of the reflection spectra of all representative two-by-two patterns, any halftone print can by tiled into representative two-by-two patterns and the reflectance of specific areas can be predicted thanks to Eqs. (20) or (21), where the $n$-value is 
generally similar for single ink or multiple ink halftones. This $n$-value can therefore be learned from a set of single ink halftones, printed on paper and on different solid colorants, for example the halftones shown in Figure 3, rows $\mathrm{C}$ and $\mathrm{F}$.

Experience has shown that for different halftone screen frequencies, the optimal $n$-values used for the assembly of representative two-by-two square patterns according to Eqs. (20) or (21) may vary. Since the representative two-by-two square patterns already incorporate the optical dot gain induced by its 4 neighboring pixels, these optimal $n$-values are generally smaller than the ones used for predicting the reflectance of the same halftones with the YuleNielsen modified spectral Neugebauer model presented in Section 3.

\section{Comparing the prediction accuracy of the models}

In order to compare the prediction accuracy of the different prediction models, several tests were carried out from various patch sets printed with different printing systems, different inks, on different printing supports and with different halftoning methods. The tested models are the Cellular Yule-Nielsen model (depending on a parameter $n$ ), the Yule-Nielsen model (also depending on a parameter $n$ ), the Clapper-Yule model and the low-scattering ClapperYule model (depending on a parameter $b$ ), and the two-by-two dot centering model. The Clapper-Yule and Yule-Nielsen models are calibrated using the superposition-dependent ink spreading assessment method. The Cellular Yule-Nielsen model is calibrated with a single ink spreading curve per ink and per subdomain. The two-by-two dot centering model is calibrated by printing and measuring the reflectances of the 1072 representative two-by-two patterns.

For the Yule-Nielsen and Clapper-Yule models, we first show some general tendencies. We then illustrate by various examples the specific influence of the paper, the halftone screen frequency and the measuring geometry on the prediction accuracy of the models. Prediction accuracy is assessed by the average CIELAB $\triangle E 1994$ value computed between the predicted and measured spectral reflectances for each patch of a large color patch set. This $\Delta E$ value, denoted $\Delta E_{94}$, is obtained by converting reflectances to CIE-XYZ tristimulus values calculated with a D65 illuminant for the $2^{\circ}$ standard observer, and then into CIELAB color coordinates using as white reference the spectral reflectance of the unprinted paper illuminated with the D65 illuminant. We generally consider that the prediction accuracy is good when the average $\Delta E_{94}$ value computed over a given patch set is below the just noticeable color distance, i.e. $\Delta E_{94}<1$. In order to assess the maximum deviation between prediction and measurement while excluding potential aberrant deviations due to printing or measuring defects, we also specify the 95-percentile $\Delta E_{94}$ value, indicating the largest deviations while excluding the worst $5 \%$ predictions.

Spectral measurements were carried out with the X-Rite $i 1$ spectrophotometer which illuminates the sample at $45^{\circ}$ from its normal and captures the reflected radiance in the normal direction $\left(45^{\circ}: 0^{\circ}\right.$ geometry according to the standard CIE notation), the X-Rite Color 
i7 spectrophotometer which illuminates the sample with Lambertian light and captures the reflected radiance at $8^{\circ}$ from the normal by including (di: $8^{\circ}$ geometry) or excluding (de: $8^{\circ}$ geometry) the specularly reflected light at the print-air interface.

General tendencies. Let us first compare the prediction accuracy of the models from set of colors printed with different printing processes: electrophotography, inkjet, and offset lithography. Electrophotographic samples were made of $125 \mathrm{CMY}$ halftones generated at 100 lpi, printed on Canon MP101 $170 \mathrm{~g} / \mathrm{m}^{2}$ matte paper with the Xerox Phaser 6360DN printer (densities of the cyan, magenta and yellow inks were respectively 1.31, 1.04 and 1.16), measured using a $45^{\circ}: 0^{\circ}$ geometry. Inkjet samples were made of 125 halftones also generated at 100 lpi, printed on the same paper, with the Canon Pixma Pro9500 Mark II inkjet printer, measured using a di: $8^{\circ}$ geometry (densities of the cyan, magenta and yellow inks were respectively $0.82,0.57$ and 0.72 ). Offset samples were made of $729 \mathrm{CMY}$ halftone patches generated at 150 lpi and at 75 lpi on coated paper with a Komori offset press (densities of the cyan, magenta and yellow inks were respectively 1.47, 1.28 and 1.43), measured using a $45^{\circ}: 0^{\circ}$ geometry. The prediction accuracy of the models is presented in part A of Table 3.

The prediction accuracy may depend on many factors that are studied in detail in the following paragraphs. These examples are representative of the performance of the models. In inkjet printing, excellent prediction accuracy is achieved with the four models provided the ink density is not too high (see below about the influence of the ink densities). Except for the Clapper-Yule model, which does not work well at low screen frequencies, good prediction accuracy is also achieved in offset printing with typical ink densities. In electrophotography printing, the prediction accuracy is lower. This is due to the fact that colored toners are more scattering than the inks used in inkjet and offset printing. The Cellular Yule-Nielsen model is generally the most accurate prediction model due to the fact that its calibration set comprises the reflectances of all superposition variations of full tones and 50\% surface coverage halftones.

Influence of the ink densities. The densities of inks have a strong influence on the prediction accuracy of the models. A high density induces a very low reflectance of the solid ink patch in the wavelength domain where the ink is the most absorbing. Since solid inks are used for the calibration of the model and since the accuracy of the spectrophotometer may be poor at low reflectance levels, the overall performance of the calibrated model may be negatively affected. In order to illustrate this, we used a same set of 125 halftones generated at 100 lpi, printed on the same Canon MP101 $170 \mathrm{~g} / \mathrm{m}^{2}$ matte paper with the same Canon Pixma Pro9500 Mark II inkjet printer, and measured with the same instrument based on a di: $8^{\circ}$ geometry. In one case, the inks were printed at the maximal drop size (the densities for cyan, magenta and yellow inks were respectively 1.37, 1.25 and 1.24) and in the other case, the drop size was divided by 2 (densities $0.82,0.57$ and 0.72 ). As can be seen in part B of Table 
3, the prediction accuracy of all models is considerably increased when the ink densities are reduced. With the highest ink densities, only the Cellular Yule-Nielsen model provides satisfying predictions thanks to the significant number of primaries incorporating superpositions of $0 \%$ and $50 \%$ ink surface coverage halftones. These primaries reduce the influence of the noisy full tone reflectances.

Influence of the paper. It is known that the paper properties have a strong influence on the color rendering of prints. The prediction accuracy of the models also depends on the type of paper. This is illustrated by part $\mathrm{C}$ of Table 3 where the Cellular-Yule Nielsen model, the Yule-Nielsen model and the Clapper-Yule model were tested on a same set of 125 color patches on different papers: Canon MP101 $170 \mathrm{~g} / \mathrm{m}^{2}$ matte paper, HP Premium $240 \mathrm{~g} / \mathrm{m} 2$ glossy paper and common $80 \mathrm{~g} / \mathrm{m}^{2}$ office paper. All the halftones were generated at $100 \mathrm{lpi}$ and printed with the Canon Pixma Pro9500 Mark II inkjet printer with the same printing options as above. They were measured under a diffuse illumination by capturing the reflected light at $8^{\circ}$ including the specular reflection (di: $8^{\circ}$ geometry). The densities for the cyan, magenta and yellow inks were respectively: 1.37, 1.25 and 1.24 for the Canon matte paper, 1.84, 1.6 and 1.33 for the HP glossy paper, and 1.06, 1.01 and 1.07 for the office paper. We again observe that the Cellular Yule-Nielsen model is more accurate than the Yule-Nielsen and Clapper-Yule models. However, these two last models perform well for the office paper.

In a second experiment, a set of 40 patches was generated at 120 lpi and printed with the Canon Pixma Pro9500 inkjet printer with the same printing options, then measured using a di: $8^{\circ}$ geometry. The calibration of the Yule-Nielsen and Clapper-Yule models was performed with $8+12=20$ patches. The calibration of the Cellular Yule-Nielsen was performed with $27+8=35$ patches. The printed papers were: the same common office paper $80 \mathrm{~g} / \mathrm{m}^{2}$ as the one presented in part D of Table 3 (densities $0.47,0.37$ and 0.6 for the cyan, magenta, respectively yellow inks), a non-fluorescent noncalendered $80 \mathrm{~g} / \mathrm{m}^{2}$ paper called Biotop, known to be quite porous (ink densities $0.45,0.35$ and 0.56), and a Canson $90 \mathrm{~g} / \mathrm{m}^{2}$ tracing paper in front of a perfectly black background (ink densities $0.84,0.81$ and 0.86 ). The papers' transmittances between 550 and $600 \mathrm{~nm}$ gives an idea of their opacity: it is around 0.19 for the Biotop paper, 0.16 for the office paper, and 0.74 for the tracing paper. The tracing paper is much more translucent, i.e. much less scattering, than the other papers. As shown in part D of Table 3, the prediction accuracy of the Yule-Nielsen and Clapper-Yule models is excellent for the office and Biotop papers, which is expected due to the low ink densities, but it is very poor for the low scattering tracing paper despite its relatively low ink density values.

Influence of the halftone screen frequency. When the halftone frequency increases, the optical dot gain increases, because the propagation distance of light due to scattering in the paper, which is constant for a given paper medium, becomes larger in comparison with the halftone period. In order to see how the prediction accuracy and the parameters of the models 
vary according to the halftone screen frequency, a same set of 125 color patches were printed at 50, 75, 100 and 125 lpi with the same Epson P50 inkjet printer on the same Canon MP101 $170 \mathrm{~g} / \mathrm{m} 2$ matte paper. The densities of the cyan, magenta and yellow inks were respectively $0.96,0.78$ and 1.05. The samples are measured according to the $45^{\circ}: 0^{\circ}$ geometry. The prediction accuracy of the Cellular Yule-Nielsen model, the Yule-Nielsen model, the ClapperYule model and the low scattering Clapper-Yule model are presented in part E of Table 3.

In the case of the Cellular Yule-Nielsen model, excellent prediction accuracy is achieved for the four tested frequencies. It is interesting to notice the increase of the optimal $n$ value as a function of the screen frequency, which is consistent with the correlation of the $n$ value and the degree of optical dot gain. The same comments apply to the Yule-Nielsen model: the prediction accuracy, although slightly lower than for the Cellular Yule-Nielsen model, is still excellent, and the optimal $n$ value increases with the halftone screen frequency.

The Clapper-Yule model assumes a large propagation distance of light within the paper and is therefore more adapted to high halftone screen frequencies: its predictive performance increases with the halftone screen frequency. At low frequencies, the low scattering ClapperYule model performs better and achieves comparable prediction accuracy as the Yule-Nielsen model. This improvement is due to the weighted average between the Clapper-Yule model adapted to high screen frequencies and the Neugebaueur model adapted to low screen frequencies since it assumes no lateral propagation of light within the paper. This is confirmed by the fact that at low frequencies, the optimal $b$ weighting factor is higher, thus giving more weight to the Neugebauer model.

Influence of the measuring geometry. The measuring geometry may have an influence on the predictive performance of the model due to the specular reflection by the surface, or possibly due to the colored sheen appearing as a consequence of optical effects at the surface of the inks. In order to illustrate the difference in predictive performance of the models according to the measurement geometry, we printed $125 \mathrm{CMY}$ color patches at 150 lpi with the Canon Pixma pro9500 II inkjet printed on Canon PP201 260 g/m2 glossy coated paper and measured them with the X-rite Color i7 spectrophotometer in diffuse: $8^{\circ}$ geometry with specular component included (di: $8^{\circ}$ geometry) or excluded (de: $8^{\circ}$ geometry) as well as the $\mathrm{X}$ Rite il spectrophotometer based on the $45^{\circ}: 0^{\circ}$ geometry. Under specular visual observation, these samples display a striking reddish sheen on patches containing a high amount of cyan ink. The prediction accuracies of the Cellular Yule-Nielsen model, the Yule-Nielsen model and the Clapper-Yule model are illustrated in part F of Table 3. Since the halftones are printed at a high screen frequency, the low scattering Clapper-Yule model has an optimal $b$ value of 0 and is therefore identical to the Clapper-Yule model. When the specular component is captured by the detector, i.e. with the di: $8^{\circ}$ geometry, the Yule-Nielsen, Clapper-Yule and low scattering Clapper-Yule models have a lower accuracy than with the other measuring 
geometries. The best accuracy is achieved with the $45^{\circ}: 0^{\circ}$ geometry for which the specular reflection, and therefore the sheen, is less apparent.

Table 3. Predictive performance of the models according to various parameters.

\begin{tabular}{lllll}
\hline \hline & $\begin{array}{c}\text { Cellular Yule-Nielsen } \\
\text { model }\end{array}$ & Yule-Nielsen model & $\begin{array}{c}\text { Clapper-Yule } \\
\text { model }\end{array}$ & $\begin{array}{c}\text { Low scattering } \\
\text { Clapper-Yule model }\end{array}$ \\
\hline A) Printing process (sets of 125 halftones for electrophotography and inket, and of 729 halftones for offset) \\
\hline $\begin{array}{l}\text { Electrophotography } \\
(150 \text { lpi) }\end{array}$ & $0.89(2.1)^{a}[n=6.5]$ & $1.04(2.2)[n=6.5]$ & $1.28(2.5)$ & $1.28(2.5)[b=0]$ \\
Inkjet (100 lpi) & $0.15(0.5)[n=5]$ & $0,22(0,5)[n=5]$ & $0.32(0.7)$ & $0.27(0.6)[b=0.1]$ \\
Offset (150 lpi) & $0.76(1.6)[n=2]$ & $0.88(1.8)[n=2]$ & $0.98(1.8)$ & $0.98(1.8)[b=0]$ \\
Offset (75 lpi) & $0.91(1.9)[n=1.5]$ & $0.64(1.3)[n=1.5]$ & $1.26(2.47)$ & $0.81(1.71)[b=0.5]$ \\
\hline
\end{tabular}

B) Ink density (sets of 125 CMY halftones, 100 lpi)

\begin{tabular}{llllll}
\hline Cyan: & $\mathrm{d}_{\mathrm{c}}=1.37$ & & & & \\
Magenta: & $\mathrm{d}_{\mathrm{m}}=1.25$ & & & & \\
Yellow: & $\mathrm{d}_{\mathrm{y}}=1.24$ & $0.56(1.4)[n=10]$ & $1.14(2,4)[n=10]$ & $1.35(3.4)$ & $1.35(3.4)[b=0]$ \\
Cyan: & $\mathrm{d}_{\mathrm{c}}=0.82$ & & & & \\
Magenta: & $\mathrm{d}_{\mathrm{m}}=0.57$ & & & & \\
Yellow & $\mathrm{d}_{\mathrm{y}}=0.72$ & $0.15(0.5)[n=5]$ & $0.22(0,5)[n=5]$ & $0.32(0.7)$ & $0.27(0.6)[b=0.1]$ \\
\hline
\end{tabular}

C) Type of paper (sets of 125 CMY halftones, 100 lpi)

\begin{tabular}{llll}
\hline $\begin{array}{l}\text { Canon MP101 } 170 \mathrm{~g} / \mathrm{m}^{2} \\
\text { matte paper }\end{array}$ & $0.56(1.4)[n=10]$ & $1.14(2,4)[n=10]$ & $1.35(3.4)$ \\
$\begin{array}{l}\text { HP Premium } 240 \mathrm{~g} / \mathrm{m}^{2} \\
\text { paper }\end{array}$ & $0.64(1.7)[n=7.3]$ & $1.52(3,1)[n=7.3]$ & $2.06(4.1)$ \\
$80 \mathrm{~g} / \mathrm{m}^{2}$ office paper & $0.70(2.0)[n=10]$ & $1.08(3,0)[n=10]$ & $1.12(2.9)$ \\
\hline
\end{tabular}

D) Type of paper (sets of 40 color halftones, 120 lpi)

\begin{tabular}{llll}
$80 \mathrm{~g} / \mathrm{m}^{2}$ office paper & $\mathrm{d}_{\mathrm{c}}=0.47, \mathrm{~d}_{\mathrm{m}}=0.37, \mathrm{~d}_{\mathrm{y}}=0.6$ & $0.70(1.1)^{a}[n=10]$ & $0.50(0.8)$ \\
$80 \mathrm{~g} / \mathrm{m}^{2}$ Biotop non- & & & \\
fluorescing paper & $\mathrm{d}_{\mathrm{c}}=0.45, \mathrm{~d}_{\mathrm{m}}=0.35, \mathrm{~d}_{\mathrm{y}}=0.56$ & $0.65(1.1)[n=7.3]$ & $0.44(0.7)$ \\
$90 \mathrm{~g} / \mathrm{m}^{2}$ tracing paper & $\mathrm{d}_{\mathrm{c}}=0.84, \mathrm{~d}_{\mathrm{m}}=0.81, \mathrm{~d}_{\mathrm{y}}=0.86$ & $2.99(4.6)[n=10]$ & $3.03(4.7)$ \\
\hline
\end{tabular}

E) Halftone screen frequency (sets of $125 \mathrm{CMY}$ halftones)

\begin{tabular}{lllll}
\hline 50 lpi & $0.42(1.13)[n=2]$ & $0.53(1.02)[n=2]$ & $1.01(1.96)$ & $0.52(1.05)[b=0.5]$ \\
$75 \mathrm{lpi}$ & $0.35(1.07)[n=3]$ & $0.46(0.94)[n=2]$ & $0.70(1.38)$ & $0.49(1.12)[b=0.3]$ \\
$100 \mathrm{lpi}$ & $0.42(1.38)[n=6]$ & $0.49(1.01)[n=4]$ & $0.62(1.34)$ & $0.58(1.18)[b=0.1]$ \\
$125 \mathrm{lpi}$ & $0.37(1.23)[n=9]$ & $0.42(0.88)[n=5]$ & $0.45(0.96)$ & $0.45(0.96)[b=0]$ \\
\hline \multicolumn{5}{l}{ F) Measuring geometry (sets of $125 \mathrm{CMY}$ halftones) } \\
\hline di: $8^{\circ}$ & $0.35(0.8)[n=3.8]$ & $1.01(1.9)[\mathrm{n}=3.8$ & $0.92(2.0)$ & \\
de: $8^{\circ}$ & $0.38(1.0)[n=10]$ & $0.73(1.4)[n=10$ & $0.93(1.9)$ & \\
$45^{\circ}: 0^{\circ}$ & $0.42(1.1)[n=3.4]$ & $0.52(1.2)[n=3.4$ & $0.75(1.5)$ & \\
\hline
\end{tabular}

${ }^{a}$ CIELAB $\Delta \mathrm{E}_{94}$ values: Average (95-percentile) over the whole patch set.

Four-ink printing. The models were also tested with three sets of 625 four-ink halftones all printed on Canon MP101 $170 \mathrm{~g} / \mathrm{m} 2$ matte paper, and measured with the $45^{\circ}: 0^{\circ}$ geometry. The first set was printed at 100 lpi with a Canon IP4000 inkjet printer using cyan, magenta, yellow, and black inks (CMYK). The second set was printed at 120 lpi with a Canon Pro9500 
inkjet printer using cyan, magenta, yellow inks and a custom green ink. The third set was also printed at 120 lpi with a Canon Pro9500 inkjet printer, using blue, magenta and yellow and orange inks (BMYO).The average and 95-percentile $\Delta \mathrm{E}_{94}$ values yielded by the four models are given in Table 4.

The prediction accuracy of the models in CMYK printing is comparable to the one in CMY printing if we compare the $\Delta \mathrm{E}_{94}$ values given in the first row of Table 4 with those presented in the first row of part $\mathrm{C}$ in Table 3 where the same paper and similar ink drop sizes were used. For the two other patch sets, the $\Delta \mathrm{E}_{94}$ values can be compared with the ones presented in the second row of part B in Table 3 where the drop size was comparable: although slightly lower, the prediction accuracy is as excellent with four inks as with three inks. This shows that even in the case of non-standard inks, the models for three or four inks offer an excellent prediction accuracy.

Table 4. Predictive accuracy of models for 4-ink halftones, in terms of CIELAB $\Delta \mathrm{E}_{94}$ differences between predicted color and measured color.

\begin{tabular}{lccc}
\hline \hline $\begin{array}{c}\text { Ink set, halftone frequency, } \\
\text { densities }\end{array}$ & $\begin{array}{c}\text { Cellular Yule-Nielsen } \\
\text { model }\end{array}$ & Yule-Nielsen model & Clapper-Yule model \\
\hline CMYK, 100 lpi & $0.66(1.6)$ & $1.13(2.8)$ & $1.28(2.9)$ \\
$\mathrm{d}_{\mathrm{c}}=0.87, \mathrm{~d}_{\mathrm{m}}=1.37, \mathrm{~d}_{\mathrm{y}}=1.4$, & with $n=2$ & with $n=2$ & \\
CMYG, 120 lpi & $0.31(0.8)$ & $0.54(1.1)$ & $0.67(1.3)$ \\
$\mathrm{d}_{\mathrm{c}}=0.98, \mathrm{~d}_{\mathrm{m}}=0.74, \mathrm{~d}_{\mathrm{y}}=1.06$ & with $n=3$ & with $n=2$ & \\
BMYO, 120 lpi & $0.36(1.0)$ & $0.60(1.3)$ & $0.85(1.9)$ \\
$\mathrm{d}_{\mathrm{m}}=0.75, \mathrm{~d}_{\mathrm{y}}=1.0$ & with $n=6$ & with $n=4$ & \\
\hline
\end{tabular}

${ }^{a}$ Average (95-percentile) CIELAB $\Delta \mathrm{E}_{94}$ value over 625 color patches.

Basic versus superposition-dependent ink spreading assessment method. Regarding the ink spreading assessment method, compared with the basic method, the superpositiondependent method improves considerably the prediction accuracy of the models. This is illustrated by the diagram of Figure 9 where the coordinates of a point represent the average $\Delta \mathrm{E}_{94}$ values obtained with the Clapper-Yule model or the Yule-Nielsen model calibrated using the basic method (in abscissa) and with the superposition-dependent method (in ordinate) on the previously presented patch sets. Since all points are below the dotted line of slope 1, for all considered cases, better prediction accuracy is achieved with the superposition-dependent method. In a number of cases, the improvement in prediction accuracy is spectacular. 


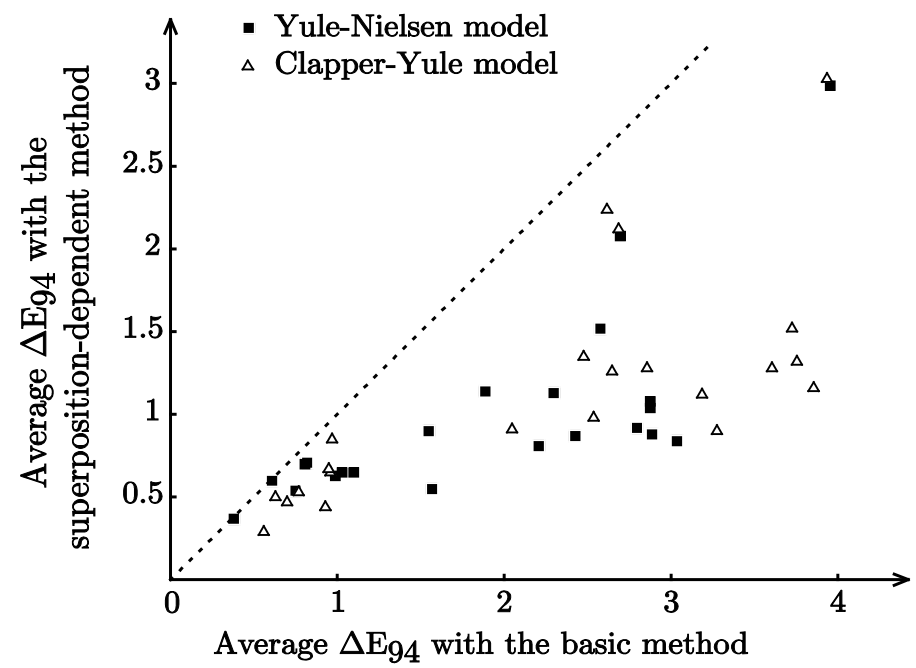

Figure 9. Average $\triangle E 94$ values between the measured and predicted spectral reflectances of 22 halftones when using as ink spreading calibration the BIS method (abscissa) or the SDIS method (ordinate).

\section{Prediction accuracy of the two-by-two dot centering model}

Table 4 shows the prediction accuracy of the two-by-two dot centering prediction model in the case of CMY 3-ink prints, for the prediction of mutually rotated clustered dot halftones printed at frequencies of $75 \mathrm{lpi}, 100 \mathrm{lpi}$ and $125 \mathrm{lpi}$ and for the prediction of diffuse dither halftones (same sets of 125 halftones as in Table 2). The patches were printed at 600 dpi with a Canon 9500 inkjet printer on MP101 paper and measured according to a $\left(45^{\circ}: 0^{\circ}\right)$ geometry. The calibration set comprises the 1072 printed patches forming the two-by-two representative patterns.

Table 5. Prediction accuracy of the two-by-two dot centering model ${ }^{a}$

\begin{tabular}{lcc}
\hline \hline Ink set, halftone frequency & Two-by-two dot centering & Yule-Nielsen model \\
\hline Clustered dot 75 lpi & $0.83(1.48)^{b}$ & 0.43 \\
with $n=2.2$ & with $n=5.9$ \\
Clustered dot 100 lpi & $0.67(1.22)$ & 0.42 \\
& with $n=3.8$ & with $n=14$ \\
Clustered dot 125 lpi & $0.61(1.10)$ & 0.60 \\
Diffuse dither & with $n=6.8$ & with $n=14$ \\
& $0.71(1.44)$ & 1.08 \\
\hline
\end{tabular}

${ }^{a}$ with a calibration set of 1072 color patches used for all two-by-two dot centering predictions (max $n$-value is 14; larger $n$-values have only an impact on the third decimal of the color difference). Solid cyan, magenta and yellow patches have densities of $0.97,0.83$ and 1.06 respectively, according to the DIN 16536-2 density standard.

\footnotetext{
${ }^{b}$ Average (95-percentile) CIELAB $\Delta \mathrm{E}_{94}$ value over 125 color patches.
} 
As can be seen, the two-by-two dot centering model is accurate, but in the case of clustereddot halftones, slightly less precise than the Yule-Nielsen model accounting for ink spreading in all superposition conditions. The two-by-two dot centering model has the advantage of being extremely flexible and of offering good prediction accuracies for non-standard halftones whose colorant surface coverages cannot be calculated according to the Demichel equations (1) or (18).

\section{Conclusions}

The interaction of light and printed color halftones is a complex non-linear phenomenon. Over the years, prediction models were developed that are able to predict the spectral reflectance of halftones printed at high, middle or low screen frequencies with classical or custom clustered-dot or dispersed-dot screens, provided these hafltones are printed on the same support, with the same inks and with the same printer as the ones used for the calibration of the prediction model. Today, these models are often used in inverse mode, i.e. nominal ink dot surface coverages are fitted so as to obtain a reflection spectrum or a color as close as possible to the desired one. In inverse mode, the prediction models are very useful for filling the tables used in color reproduction workflows [22] which express the relationship between desired colors to inks surface coverages.

Through the different experiments carried out for this study, one can conclude that the most accurate prediction model classical clustered-dot or error diffusion halftones is the Cellular Yule-Nielsen model, mainly because it is calibrated from a larger set of halftones. This is true whatever are the printing process, the halftone screen frequency, and the printing support. The Yule-Nielsen model, the Clapper-Yule model and the Low Scattering Clapper-Yule model are less accurate but need fewer halftones for their calibration. The number of halftones that can be reasonably printed in a given application is therefore determinant for the selection of the model. Another determinant selection criterion is the possibility to predict the color of halftones when the ink thickness or the illumination or viewing conditions vary: the ClapperYule and low scattering Clapper-Yule models should be preferred in this case since they incorporate explicitly the illumination and observation geometry and the transmittance of the inks [23].

The limitation of current predictive models is their inability to predict the reflectance of superimposed solid inks knowing the reflectances of the contributing individual solid ink patches and of the unprinted paper. Several approaches exist, but do not seem to offer high prediction accuracies [24], [25], [26]. Current research focuses on using spectral prediction models in multi-ink printing systems where additional objectives need to reached, e.g. minimizing metamerism [27], minimizing the visibility of the halftones [28] or minimizing the usage of inks [29]. 


\section{Acknowledgements}

The authors would like to express their gratitude to Romain Rossier, Jacques Machizaud, Vahid Babaei and Saurabh Jain for their contribution to the experiments presented in this study. This study has been partly financed by the Swiss National Science Foundation, grant 200021_143501/1.

\section{References}

[1] H. Kipphan, Handbook of Print Media: Technologies and Production Methods, Springer (2001).

[2] H.E.J. Neugebauer, "Die theoretischen Grundlagen des Mehrfarbendrucks," Zeitschrift fuer wissenschaftliche Photographie 36, 36-73, (1937), translated into English: "The theoretical basis of multicolour letterpress printing", Color Res. App. 30, 322-331 (2005).

[3] J.A.C. Yule, W.J. Nielsen, "The penetration of light into paper and its effect on halftone reproduction", Proc. TAGA 3, 65-76 (1951).

[4] J.A.S. Viggiano, "The Color of Halftone Tints”, Proc. TAGA, 647-661 (1985).

[5] R. Balasubramanian, Optimization of the spectral Neugebauer model for printer characterization, J. Electronic Imaging, 8, (2), 156-166 (1999).

[6] R.D. Hersch, F. Crété, "Improving the Yule-Nielsen modified spectral Neugebauer model by dot surface coverages depending on the ink superposition conditions", Proc SPIE. 5667, 434445 (2005).

[7] V. Babaei, R.D.Hersch, Spectral Prediction of Juxtaposed Halftones Relying on the Two-bytwo Dot Centering Model, Journal of Imaging Science and Technology, 57 (4), article 040501, 9 pages (2013)

[8] S. G. Wang, "Two-by-two centering printer model with Yule-Nielsen equation," IS\&T International Conference on Digital Printing Technologies, 302-305 (1998).

[9] K. J. Heuberger, Z. M. Jing, and S. Persiev, Color Transformations and lookup tables, in Proc. 1992 TAGA/ISCC, 863-881 (1992).

[10] F. R. Clapper and J. A. C. Yule, "The Effect of Multiple Internal Reflections on the Densities of Halftone Prints on Paper", J. Opt. Soc. Am. 43, 600-603 (1953).

[11] R. Rolleston, R. Balasubramanian, Accuracy of various types of Neugebauer model, Proc. IS\&T and SID Color Imaging Conference: Transforms and Transportability of Color, 32-37 (1993).

[12] D. Wyble, R.S. Berns, A critical review of spectral models applied to binary color printing, Color Research and Applications, 25 (1), 4-19 (2000)

[13] J.S. Arney, P.G. Engledrum, H. Zeng, An expanded Murray-Davies model of tone reproduction in halftone imaging, Journal of Imaging Science and Applications, 39, 502-508 (1995)

[14] M.E. Demichel, Procédés 26 (3), 17-21 (1924).

[15] F. R. Ruckdeschel and O. G. Hauser, "Yule-Nielsen effect in printing: a physical analysis", Appl. Opt. 17, 3376-3383 (1978).

[16] R. Rossier,T. Bugnon, R.D. Hersch, Introducing ink spreading within the cellular Yule-Nielsen modified Neugebauer model, in Proc. IS\&T/SID's 18th Color Imaging Conference, 295-300 (2010). 
[17] R.D. Hersch, M. Hébert, "Interaction between Light, Paper and Color Halftones: Challenges and Modelization Approaches," Proc. IS\&T/CGIV Third European Conference on Color in Graphics, Imaging and Vision, 1-7 (2006).

[18] R.D. Hersch, P. Emmel, F. Collaud, F. Crété , "Spectral reflection and dot surface prediction models for color halftone prints", J. Electronic Imaging 14, 33001-12 (2005).

[19] V. Babaei, R. Rossier, R.D. Hersch, "Reducing the number of calibration patterns for the twoby-two dot centering model",Color Imaging XVII: Displaying, Processing, Hardcopy, and Applications, Proc. SPIE 8292, 829208:1-9 (2012).

[20] T. Bugnon, M. Brichon, R.D. Hersch, "Model-based deduction of CMYK surface coverages from visible and infrared spectral measurements of halftone prints," Proc. SPIE 6493, 649310 (2007).

[21] S.G.Wang, Feedback for printer Color Calibration, IS\&T International Conference on Digital Printing Technologies, 327-330 (1999).

[22] J.M. Lammens, Applications of Color Science and Technology in Digital Printer Research \& Development, Proc. IS\&T $4^{\text {th }}$ European conference on colour in graphics, imaging, and vision (CGIV), 1-6, (2008)

[23] R. D. Hersch, et al., Deducing ink thickness variations by a spectral prediction model, Color Research and Application, Vol. 34, No. 6, 432-442 (2009)

[24] J.P. Van de Cappelle, B. Meireson, A new method characterizing colour printing devices, in Color Imaging: Vision and Technology, Eds. L.W. MacDonald, M.R. Luo, Wiley, 179-188 (1999)

[25] D.-Y. Tzeng and R. S. Berns. Spectral-Based Ink Selection for Multiple-Ink Printing II. Optimal Ink Selection. In Proc. IS\&T 7th Color Imaging Conference (CIC), 182-187 (1999).

[26] K. Deshpande, P. Green, A simplified method of predicting the colorimetry of spot color overprints, In Proc. IS\&T 18th Color Imaging Conference (CIC), 213-216 (2010).

[27] P. Urban, R. S. Berns, Paramer Mismatch-Based Spectral Gamut Mapping, IEEE Trans. Image Processing, 20 (6), 1599-1610 (2011)

[28] S. Chosson, R.D. Hersch, Visually-based color space tetrahedrizations for printing with custom inks, Proc. SPIE, Color Imaging: Device-Independent Color, Color Hardcopy, and Graphic Arts VI, Proc. SPIE 4300, 81-92 (2001).

[29] M. V. Ortiz Segovia, N. Bonnier and J. P. Allebach, "Ink-saving strategy based on document content characterization and halftone textures", Color Imaging XVII: Displaying, Processing, Hardcopy, and Applications, Proc. SPIE 8292, paper 829211, 13 pages (2014). 